\title{
Melatonin for supportive and selective cancer therapeutics
}

Keywords: melatonin, immune system, breast cancer, inflammation, brain

\section{Commentary}

Melatonin has numerous potential effects for the immune system. ${ }^{1}$ Melatonin is a powerful antioxidant and free radical scavenger that helps fight inflammation. ${ }^{2}$ In fact, because melatonin is essential to the immune system, a lack of melatonin causes thymic atrophy, a key component of the immune system. Melatonin can also delay the aging of the brain. ${ }^{3}$

This hormone also interferes with the supply of new blood for the rapid growth of the tumor and angiogenesis. ${ }^{4}$ Melatonin increases the effectiveness of cancer chemotherapy and reduces toxicity. ${ }^{5}$ Current research suggests that melatonin deficiency may present some nuisance biological shortcomings, such as high levels of inflammation, weakened immune systems, and increased risk of cancer. One of the obvious ways to reduce the natural melatonin production of the human body is to expose to artificial light at night, even for a short time. ${ }^{6}$ Several studies have found that night shift workers have a higher incidence of cancer, especially breast cancer. ${ }^{7}$ Although supplements can help, it is much more beneficial for the body to produce melatonin on its own. This natural way of obtaining "perfect" melatonin capacity, is a way not overflowing and not wasting away. Because the human body uses feedback circuitry to adjust the right dose, it is possible to obtain the most adequate amount of melatonin through natural methods. Hence, our research suggests that due to the importance of sleep in improving the natural secretion melatonin, the increase of physiological sleep will have beneficial effects on the immune system via melatonin secretion. However, as mentioned above, if there is a reason one cannot naturally stretch melatonin, clinicians can consider supplements, but using the suggestions outlined above is still a much wiser method. ${ }^{8,9}$

\section{Acknowledgement}

None.

\section{Conflicts of interest}

The author declared that there are no conflicts of interest.

\section{References}

1. Elmahallawy EK, Luque JO, Aloweidi AS, et al. Potential Relevance of Melatonin Against Some Infectious Agents: A Review and Assessment of Recent Research. Curr Med Chem. 2015;22(33):3848-3861.
Volume 2 Issue 2 - 2018

\author{
Seung Chan Kim \\ Medical School \& College of Medicine, Yonsei University, Korea \\ Correspondence: Seung Chan Kim, Medical School \& College \\ of Medicine, Yonsei University, Yonsei-ro 50, Shinchon-dong, \\ Seodaemoon-gu, Seoul, Republic of Korea, Korea, Tel +8226 \\ 2452 835, Email synergy@yonsei.ac.kr, synedit@gmail.com
}

Received: June 01, 2017 | Published: April 03, 2018

2. Chen Y, Zhao Q, Sun Y, et al. Melatonin inducesanti-inflammatory effects via endoplasmic reticulum stress in RAW264.7macrophages. Mol Med Rep. 2018;17(4):6122-6129.

3. Tan DX, Xu B, Zhou X, et al. Pineal Calcification, Melatonin Production, Aging, Associated Health Consequences and Rejuvenation of the Pineal Gland. Molecules. 2018;23(2).

4. Crooke A, Huete-Toral F, Colligris B, et al. The role and therapeutic potential of melatonin in age-related ocular diseases. $J$ Pineal Res. 2017;63(2).

5. González-González A, González A, Alonso-González C, et al. Complementary actions of melatonin on angiogenic factors, the angiopoietin/Tie2 axis and VEGF, in co-cultures of human endothelial and breast cancer cells. Oncol Rep. 2018;39(1):433-441.

6. Di Bella G, Colori B, Toscano R. Complete objective response, stable for 5 years, with the Di Bella Method, of multiple-metastatic carcinoma of the breast. Neuro Endocrinol Lett. 2017;38(6):401-407.

7. Kozaki T, Kubokawa A, Taketomi R, et al. Light-induced melatonin suppression at night after exposure to different wavelength composition of morning light. Neurosci Lett. 2016;616:1-4.

8. Xie W, Gao Q, Wang D, et al. Melatonin potentiates "inside-out" nanothermotherapy in human breast cancer cells: a potential cancer target multimodality treatment based on melatonin-loaded nanocomposite particles. Int J Nanomedicine. 2017;12:7351-7363.

9. Gatti G, Lucini V, Dugnani S, et al. Anti proliferative and pro-apoptotic activity of melatonin analogues on melanoma and breast cancer cells. Oncotarget. 2017;8 (40):68338-68353. 